Conclusions HARP and SinMod methods show a high level of agreement for assessment of global mid-ventricular transmural circumferential strain, with good reproducibility for both individual methods. Agreement is much lower for segmental measurements; poor reproducibility for segmental measurements using both techniques probably reflect user variability in identification of right ventricular septal insertion points and contour tracing.

\section{AETIOLOGICAL ROLE OF FOLATE DEFICIENCY IN CONGENITAL HEART DISEASE: EVIDENCE FROM MENDELIAN RANDOMISATION AND META-ANALYSIS}

doi:10.1136/heartjnl-2011-300198.131

V Mamasoula, T Pierscionek, D Hall, J Palomino-Doza, A Topf, T Rahman, J Goodship, B Keavney. Institute of Human Genetics, Newcastle upon Tyne, UK

Background The existence of a causal relationship between lower levels of plasma folate and congenital heart disease (CHD) remains contentious. Randomised trials of this question are not possible, in view of the known protective effect of folate against neural tube defects (NTDs). Folate fortification of flour is known to reduce the incidence of NTDs, but it is not known whether there is any effect on CHD. Clarity regarding the relationship between folate and CHD could potentially inform the practice of folate fortification. We present a genetic approach using "Mendelian randomisation" to determining the causality of folate in CHD risk.

Methods We compared genotype frequencies at the methylene tetrahydrofolate reductase (MTHFR) C677T single nucleotide polymorphism (SNP) in $1186 \mathrm{CHD}$ cases and 4168 controls. The TT genotype at MTHFR C677T is known to be associated with lower activity of MTHFR and plasma folate, and higher levels of plasma homocysteine. The effect of TT genotype on plasma folate levels is greater in conditions of folate deficiency. Thus, if lower plasma folate had a causal effect on CHD risk, a higher frequency of TT genotype among CHD cases than among healthy controls would be anticipated, and this would be expected to be more marked in conditions of folate deficiency. We placed our results in the context of a meta-analysis of all previously published studies of this question (to September 2010), which together included 1883 cases and 3069 controls in 25 studies. Thus, the combined analyses included 3069 CHD cases and 7271 controls. We used randomeffects models to combine the data. We conducted sensitivity analyses to examine folate fortification of flour as a potential source of heterogeneity.

Results The primary genotyping data in 1186 cases and 4168 controls revealed a trend towards increased risk with the TT genotype, but this did not reach statistical significance (OR 1.15 (95\% CI 0.94 to 1.40$)$ ). Combination of our primary data with previous studies, however, revealed association in the larger dataset (OR 1.45 (95\% CI 1.12 to 1.89 ); $\mathrm{p}=0.005$ ). The population attributable fraction for the TT genotype was $3 \%$ of CHD. There was no evidence of publication bias among the contributing studies. We discovered folate fortification status to be a significant source of heterogeneity. Studies conducted in countries with mandatory folate fortification showed no effect of C677T genotype on CHD risk (OR 0.96 (95\% CI 0.64 to 1.44)), whereas studies conducted in countries without mandatory fortification showed a significant effect of genotype (OR 1.63 (95\% CI 1.19 to 2.25)). These ORs were significantly different from each other $(p=0.032)$.

Conclusions We demonstrate genetic evidence in favour of a causal relationship between plasma folate and $\mathrm{CHD}$. The absence of a genetic association in countries practicing folate fortification suggests that fortification largely abrogates the risk of $\mathrm{CHD}$ attributable to folate deficiency.

\section{NON-SYNONYMOUS SMAD6 MUTATIONS IMPAIRED INHIBITION OF BMP SIGNALLING IN PATIENTS WITH CONGENITAL CARDIOVASCULAR MALFORMATION}

doi:10.1136/heartjnl-2011-300198.132

${ }^{1} \mathrm{H}$ L Tan, ${ }^{1} \mathrm{E}$ A Glen, ${ }^{1} \mathrm{~A}$ L Topf, ${ }^{1} \mathrm{D} \mathrm{H}$ Hall, ${ }^{2} \mathrm{~J} \mathrm{~J}$ 0'Sullivan, ${ }^{2} \mathrm{~L}$ Sneddon, ${ }^{2} \mathrm{C}$ Wren, ${ }^{3} \mathrm{P}$ Avery, ${ }^{4} \mathrm{R} \mathrm{J}$ Lewis, ${ }^{5} \mathrm{P}$ ten Dijke, ${ }^{1} \mathrm{H} \mathrm{M}$ Arthur, ${ }^{1} \mathrm{~J} \mathrm{~A}$ Goodship, ${ }^{1} \mathrm{~B} \mathrm{D}$ Keavney. ${ }^{1}$ Institute of Human Genetics, Newcastle University, Newcastle upon Tyne, UK; ${ }^{2}$ Freeman Hospital, Newcastle upon Tyne, UK; ${ }^{3}$ School of Mathematics \& Statistics, Newcastle University, Newcastle upon Tyne, UK; ${ }^{4}$ Institute for Cell and Molecular Biosciences, Newcastle University, Newcastle upon Tyne, UK; ${ }^{5}$ Department of Molecular Cell Biology and Center for Biomedical Genetics, Leiden University, Leiden, UK

Introduction Congenital cardiovascular malformation (CVM) exhibits familial predisposition but the specific genetic factors involved are unknown. Bone morphogenetic proteins (BMPs) regulate many processes during development, including cardiac development. Five genes of the BMP signalling were surveyed for novel variants predisposing to CVM risk. One of the genes, SMAD6, functions as an inhibitory SMAD which preferentially inhibits BMP signalling. The SMAD6 knockout mouse is characterised by cardiac valve and outflow tract defects, including aortic ossification. We hypothesised that rare functional variation in SMAD6 could predispose to congenital cardiovascular malformation (CVM).

Methods The coding regions of BMP2, BMP4, BMPR1A, BMPR2 and $S M A D 6$ were sequenced in 90 unrelated Caucasian cases of CVM The MH2 domain of SMAD6 were further sequenced in additional 346 CVM patients. Functional effects of the wild-type and variant SMAD6 proteins were expressed in $\mathrm{C} 2 \mathrm{C} 12$ cells and their capacity to inhibit ALK3 activated expression of a BMP-responsive reporter, or to inhibit osteogenic differentiation (using an alkaline phosphatase assay) was assessed.

Results We identified two novel non-synonymous variants, P415L and C484F, that were not present in 1000 ethnically-matched controls. P415L was identified in a patient with congenital aortic stenosis and C484F was identified in a patient with coarctation and calcification of the aorta. Both mutations are in evolutionarily conserved amino acid residues and are predicted to be damaging by in silico analysis. This was confirmed in functional assays as both SMAD6 variants failed to inhibit BMP signalling compared with wild-type SMAD6. The P415L mutant appeared to be hypomorphic whereas C484F appeared to be a null allele in the luciferase assay. The C484F mutant had a significantly $(p<0.05)$ lower capacity to inhibit alkaline phosphatase generation in response to BMP signalling. Conclusions This is the first time that functional mutations in SMAD6 have been described in patients with CVM, specifically those with calcific aortic malformations. Our data suggest that inadequate inhibition of BMP signalling pathway due to genetic variation in SMAD6 may be an important factor in CVM.

\section{ACTIVITY AND PSYCHOSOCIAL HEALTH IN ADOLESCENTS WITH CONGENITAL HEART DISEASE (CHD)}

doi:10.1136/heartjnl-2011-300198.133

${ }^{1} \mathrm{M} L$ Morrison, ${ }^{1} \mathrm{~A}$ J Sands, ${ }^{1,2} \mathrm{C}$ G McCusker, ${ }^{2} \mathrm{P}$ P McKeown, ${ }^{1} \mathrm{M}$ McMahon ${ }^{1} \mathrm{~J}$ Gordon, ${ }^{1} \mathrm{~B}$ G Craig, ${ }^{1}{ }^{1} \mathrm{~F}$ A Casey. ${ }^{1}$ Royal Belfast Hospital for Sick Children, Belfast, UK; ${ }^{2}$ The Queen's University of Belfast, Belfast, UK

Many patients with $\mathrm{CHD}$ are now adolescents. Like other patients with chronic illnesses they may be at higher risk of psychological/ emotional problems. Ability to exercise is an important quality of life measure and indicator of physical health. We aimed to ascertain if activity and psychosocial health were reduced in adolescents with major CHD compared to those with a minor diagnosis. Patients aged 12-20 years were identified using the Northern Ireland regional database (HeartSuite). Participants were categorised as having major or minor $\mathrm{CHD}$ and divided into four diagnostic 